\title{
LASER INDUCED FLUORESCENCE OF INDIUM VAPOUR
}

\author{
T. Weyh, A. Kopystyńska, P. Kowalczyk and C. Radzewicz \\ Institute of Experimental Physics, Warsaw University \\ Hoża 69, 00-681 Warszawa, Poland \\ (Received November 5, 1991)
}

\begin{abstract}
Indium vapour was illuminated with laser light in the wavelength range 350-372 $\mathrm{nm}$ and at $410.3 \mathrm{~nm}$. A few fluorescence bands of $\operatorname{In}_{2}$ as well as a strong background emission of indium-quartz compounds were observed. An evidence of energy transfer processes in indium vapour was found.
\end{abstract}

PACS numbers: 33.20.Kf, 34.50.Fa

\section{Introduction}

Atomic states of indium are well-known up to the ionization limit [1-3] and, for doubly excited states, even in higher energy range [1]. The information available about indium diatomic molecule is much more scarce, mostly due to experimental difficulties in producing indium vapour of sufficiently high density. Up to now $\mathrm{In}_{2}$ has been studied only in a few classical spectroscopy experiments and some unresolved bands of unidentified origin, placed in the near UV and in the violet and green parts of the visible spectrum, have been reported [4-6]. On the other hand, even the first two atomic asymptotes $5^{2} P_{1 / 2,3 / 2}+5^{2} P_{1 / 2,3 / 2}$ and $5^{2} P_{1 / 2,3 / 2}+6^{2} S_{1 / 2}$ lead to several tens of molecular states and one can expect a rich and complicated spectrum of $\mathrm{In}_{2}$ in the visible region.

In our letter we report the first observation of a laser induced fluorescence (LIF) of indium dimer. Some experimental difficulties present at cell measurements in indium vapour are also discussed. Additionally we study a variation of the effective lifetime of the $6^{2} S_{1 / 2}$ atomic state with the temperature which in our opinion gives an indirect evidence of the population of high atomic levels upon excitation of indium vapour by $410.3 \mathrm{~nm}$ resonance light. 


\section{Experimental}

Indium vapour was contained in an evacuated quartz cell heated to 1000 $1300 \mathrm{~K}$ which corresponds to atomic densities of $10^{12}-10^{14} \mathrm{~cm}^{-3}[7]$. The vapour was excited with light of a pulsed dye laser pumped by a home-made $\mathrm{XeCl}$ excimer laser. The dye laser operated with DMQ dye was tunable over the range 350-372 nm and provided pulses of about $30 \mathrm{~ns}$ duration, $0.01 \mathrm{~nm}$ spectral width and $12 \mathrm{~kW}$ peak power. The vapour could be also illuminated with light of a nitrogen laser $(\lambda=337 \mathrm{~nm}, 200 \mathrm{~kW})$, of a XeCl excimer laser $(\lambda=308 \mathrm{~nm}, 6.5 \mathrm{MW})$ and, for excitation of the $410.3 \mathrm{~nm}$ atomic resonance line, with the same dye laser as described above using the DPS dye. The fluorescence collected in the direction perpendicular to the exciting laser beam was resolved with a $0.4 \mathrm{~m}$ monochromator (Carl Zeiss Jena SPM2) to an accuracy of $0.15 \mathrm{~nm}$ and detected by a photomultiplier (FEU77) with S-20 response. The photoelectric signal was recorded by a boxcar integrator (Stanford Research System SR250) coupled to a microcomputer.

\section{Results and discussion}

We scanned the dye laser wavelength by steps of $0.05 \mathrm{~nm}$ and examined the fluorescence of indium vapour in the 280-460 nm region. Wajnkranc [4] reported several molecular absorption bands of $\mathrm{In}_{2}$ in the wavelength range covered by our excitation. To our surprise, only at the excitation wavelength $\lambda=356.9 \mathrm{~nm}$ (corresponding to $\bar{\nu}=28011 \mathrm{~cm}^{-1}$ ) we could observe a weak molecular fluorescence spectrum (Fig. 1). It consisted of three broad, unresolved bands with maxima at $345.4 \mathrm{~nm}\left(28944 \mathrm{~cm}^{-1}\right), 349.3 \mathrm{~nm}\left(28620 \mathrm{~cm}^{-1}\right)$ and $353.2 \mathrm{~nm}\left(28304 \mathrm{~cm}^{-1}\right)$. The bands appeared at temperatures about $300 \mathrm{~K}$ higher than necessary for atomic features to be seen. The approximately equal spacing of the bands suggests that they form a single vibrational progression. We tentatively ascribe the bands to a molecular transition from an unidentified excited electronic state to the ground state of $\mathrm{In}_{2}$ molecule. Then the excitation starts at least from the $\nu^{\prime \prime}=3$ level and the ground state vibrational constant is $\omega_{\mathrm{e}} \approx 320 \mathrm{~cm}^{-1}$. At present we are not able to explain the absence of LIF molecular spectra with excitation at wavelengths of absorption bands listed by Wajnkranc [4]. It must be noted that Bicchi et al. [8] have recently observed a similar fluorescence spectrum at excitation of indium vapour with the third harmonic of a Nd:YAG laser at $\lambda=355 \mathrm{~nm}$.

In the course of the experiment we noticed that after few hours operation at high temperature the quartz walls of the cell became substantially contaminated by indium. The indium-quartz compounds of unknown nature gave rise to a fluorescence background consisting of several diffuse bands and sharp lines (Fig. 2). They appeared independently of the cell temperature and displayed no resonant character to the exciting wavelength. For illumination with high power lasers $\left(\mathrm{N}_{2}\right.$ and $\left.\mathrm{XeCl}\right)$ the indium atomic resonance lines at $410.3 \mathrm{~nm}$ and $451.3 \mathrm{~nm}$ $\left(6^{2} S_{1 / 2} \rightarrow 5^{2} P_{1 / 2,3 / 2}\right.$ transitions) were additionally observed. Since the lines could be also recorded at rather low temperatures of the cell, we can exclude photodissociation of $\mathrm{In}_{2}$ molecules present in the vapour as a mechanism responsible for 


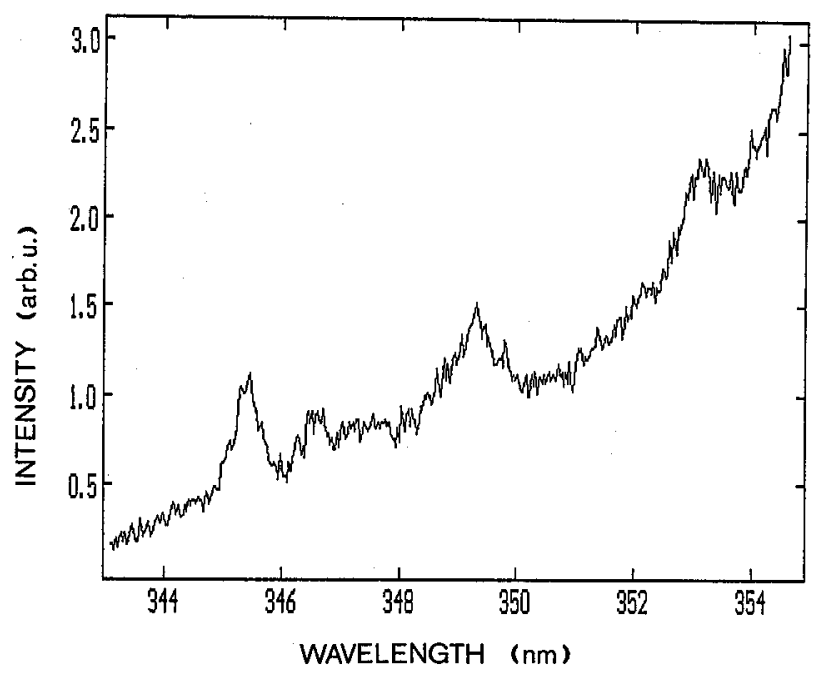

Fig. 1. Fluorescence spectrum of $\mathrm{In}_{2}$ excited with laser light at $\lambda=356.9 \mathrm{~nm}$.

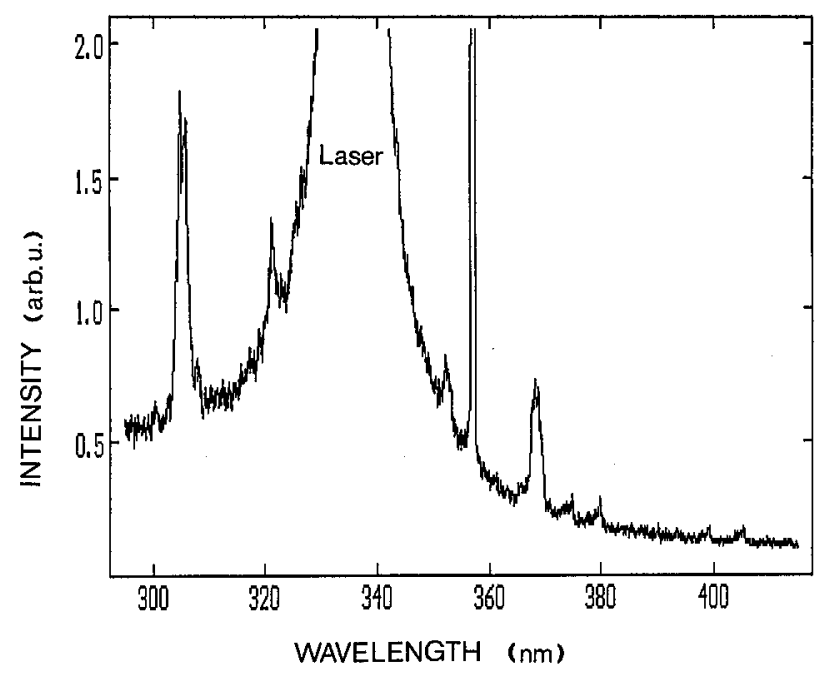

Fig. 2. Fragment of the fluorescence spectrum of a contaminated indium cell illuminated with $337 \mathrm{~nm} \mathrm{~N}_{2}$ laser light at room temperature.

their appearance. Distinct traces at the input window of the cell suggest that the focused high power laser light destroyed the indium-quartz compounds covering the inner cell walls, thus sputtering excited $6^{2} S_{1 / 2}$ atoms into the cell. The phenomenon of interaction of indium vapour with quartz is interesting in itself and deserves a separate study. On the other hand it gives rise to a rich fluorescence 
background and great care has to be taken in interpreting the spectra of indium vapour.

An additional phenomenon studied in our experiment was the change of the apparent lifetime of the $6^{2} S_{1 / 2}$ state of indium vs. vapour temperature. The $6^{2} S_{1 / 2}$ state was resonantly excited with the $410.3 \mathrm{~nm}$ line $\left(5^{2} P_{1 / 2} \rightarrow 6^{2} S_{1 / 2}\right.$ transition) and the time-resolved fluorescence at $451.3 \mathrm{~nm}\left(6^{2} S_{1 / 2} \rightarrow 5^{2} P_{3 / 2}\right.$ transition) was observed. The natural lifetime of the $6^{2} S_{1 / 2}$ state is known as $7.4 \mathrm{~ns}$ [9]. The effective lifetime measured in our experiment raised from $7.5 \mathrm{~ns}$ at $900 \mathrm{~K}$ (corresponding to atomic density $N=6.7 \times 10^{10} \mathrm{~cm}^{-3}$ ) to $19.5 \mathrm{~ns}$ at $T_{\mathrm{x}}=1210 \mathrm{~K}$ $\left(N=1.3 \times 10^{14} \mathrm{~cm}^{-3}\right)$ due to radiation trapping effects (see e.g. [10]) and started to fall down for higher temperatures. This effect, known for other dense atomic vapours [11], is explained by depopulation of the $6^{2} S_{1 / 2}$ state in collisions involving excited atoms. The interesting observation was that for temperatures exceeding $T_{\mathrm{x}}$ the decay of the $6^{2} S_{1 / 2}$ state became two-exponential, with the longer time constant of $\tau=42 \pm 15 \mathrm{~ns}$ being independent of the vapour temperature. Whereas the intensity of the short time component in the decay curve was proportional to the density $N$ of indium atoms, the long time component was proportional to $N^{2}$. We attribute it to repopulation of the $6^{2} S_{1 / 2}$ state from higher lying states of indium, possibly excited in energy-pooling collisions of two $6^{2} S_{1 / 2}$ atoms [12]. This is an indirect indication of complex energy transfer processes present in dense indium vapour irradiated with resonant laser light.

\section{References}

[1] W.R.S. Garton, K. Codling, Proc. Phys. Soc. 78, 600 (1961).

[2] M.Y. Mirza, W.W. Dudley, Proc. R. Soc. Lond. A 364, 255 (1978).

[3] C. Lorenzen, K. Niemax, K.H. Weber, Opt. Commun. 52, 178 (1984).

[4] R. Wajnkranc, Z. Phys. 104, 122 (1936); ibid. 105, 516 (1937).

[5] J.G. Winans, F.J. Davis, V.A. Leitzke, Phys. Rev. 57, 70 (1940).

[6] D.S. Ginter, M.L. Ginter, K.K. Innes, J. Phys. Chem. 69, 2480 (1965).

[7] A.N. Nesmeyanov, Vapour Pressure of the Elements, Elsevier, New York 1963.

[8] P. Bicchi, M. Meucci, A. Kopystyńska, L. Moi, private communication.

[9] A.A. Radzig, B.M. Smirnov, Reference Data on Atoms, Molecules and Ions, Springer, Heidelberg 1985.

[10] A.C. Mitchell, M.W. Zemansky, Resonance Radiation and Excited Atoms, Cambridge University Press, Cambridge 1961.

[11] A. Kopystyńska, P. Kowalczyk, Opt. Commun. 25, 351 (1978).

[12] P. Bicchi, A. Kopystyńska, M. Meucci, L. Moi, Phys. Rev. A 41, 5257 (1990). 